\title{
Application of three-dimensional printing for intraoperative guidance during liver resection of a hepatocellular carcinoma with sophisticated location
}

\author{
Jinsoo Rhu, Mi Seung Kim, Sangjin Kim, Gyu-Seong Choi, Jong Man Kim, and Jae-Won Joh \\ Department of Surgery, Samsung Medical Center, Sungkyunkwan University School of Medicine, Seoul, Korea
}

While 3D printing is adapted usefully in certain field of surgery, its application in liver surgery was limited. Here, we introduce our experience for using 3D printing for intraoperative guidance during liver resection in a case for HCC with an intrahepatic metastasis at a sophisticated location. A 50 years old male patient was diagnosed $4.7 \mathrm{~cm}-\mathrm{sized}$ hepatocellular carcinoma located on segment 3 with and an intrahepatic metastasis located on segment 8 which was between right anterior portal vein, middle hepatic vein and right hepatic vein. Since radiofrequency ablation appeared to be inappropriate, surgical resection was planned. However, the patient had a cirrhotic liver and left liver was estimated to be $47 \%$ according to volume measurement. Therefore, we planned a two-step procedure by performing left hemihepatectomy preserving the middle hepatic vein and additionally removing the intrahepatic metastasis by tumorectomy. For better guidance, we made a 3D printed model tailored for using it as a guidance during operation, and the accuracy of 3D-printed model helped the surgical team perform a safe operation. The patient underwent adjuvant proton beam therapy on the site of tumorectomy and did not experience recurrence. (Ann Hepatobiliary Pancreat Surg 2021;25:265-269)

Key Words: Hepatocellular carcinoma; 3D printing; 3D printer

\section{INTRODUCTION}

Hepatocellular carcinoma (HCC) is a malignancy originating from the liver and is one of the most common malignancies worldwide. ${ }^{1-3}$ While definite treatment for HCC is liver transplantation, most of the patient undergo other treatment modalities due to the shortage of donating organs. When the patient's liver reserve is compatible for liver resection, it can guarantee the best treatment option for those who are considered to have resectable HCC. ${ }^{4,5}$ Since the introduction of laparoscopy to the field of liver resection, minimal invasive surgery is performed widely around the world. ${ }^{6}$ As new technologies are introduced to medicine, innovative practitioners are rigorously adapting it to the field. Three-dimensional (3D) printing is one of the technologies that gained attention in the field. While $3 \mathrm{D}$ printing is adapted usefully in certain field of surgery including maxillofacial reconstruction, the application in abdominal surgery is only limited. ${ }^{7}$ Our center started to use $3 \mathrm{D}$ printing for variety of purposes and here, we introduce our experience for using 3D printing for intraoperative guidance during liver resection in a case for $\mathrm{HCC}$ with an intrahepatic metastasis at a sophisticated location.

\section{CASE}

\section{Patient}

A 50 years old male patient was diagnosed hepatocellular carcinoma located on segment 3 with and an intrahepatic metastasis located on segment 8 . Due to the sophisticated location of the intrahepatic metastasis, planning ultrasonography for radiofrequency ablation appeared to be inappropriate for ablation. The patient was a hepatitis $\mathrm{C}$ virus carrier and obtained sustained virological response after antiviral treatment. Initially, the patient had

Received: October 29, 2020; Accepted: November 26, 2020

Corresponding author: Jae-Won Joh

Department of Surgery, Samsung Medical Center, Sungkyunkwan University School of Medicine, 81 Irwon-ro, Gangnam-gu, Seoul 06351, Korea Tel: +82-2-3410-3466, Fax: +82-2-3410-1180, E-mail: jw.joh@samsung.com

Copyright (C) 2021 by The Korean Association of Hepato-Biliary-Pancreatic Surgery

This is an Open Access article distributed under the terms of the Creative Commons Attribution Non-Commercial License (http://creativecommons.org/ licenses/by-nc/4.0) which permits unrestricted non-commercial use, distribution, and reproduction in any medium, provided the original work is properly cited. Annals of Hepato-Biliary-Pancreatic Surgery • pISSN: 2508-5778 - eISSN: 2508-5859 
elevated tumor markers. Alpha fetoprotein was $41.7 \mathrm{ng} / \mathrm{ml}$ while protein induced vitamin $\mathrm{K}$ antagonist-II (PIVKA-II) was $909 \mathrm{mAU} / \mathrm{ml}$. Indocyanine green clearance test showed $13.3 \%$ of retention rate at 15 minutes.

Fig. 1 shows the radiological findings of the patient. The main tumor was located on segment 3 with a diameter of $4.5 \mathrm{~cm}$. (Fig. 1A) One centimeter-sized intrahepatic metastasis was located on segment 8 which was just superior to the right anterior portal vein after the bifurcation of right posterior portal vein. (Fig. 1B) Three-dimensional modeling was performed for better understanding of the anatomical relationship between the intrahepatic metastasis and the surrounding vascular structures. (Fig. 1C) Three-dimensional modeling was done using Materialise Mimics Medical software (Materialise, Leuven, Belgium). Volume ratio between the right and left liver was 52.1\% and $47.9 \%$, respectively. Due to the underlying liver fibrosis, performing an extended left hemihepatectomy or a left trisectionectomy was risky for the patient. Therefore, left hemihepatectomy with preservation of the middle hepatic vein and additional tumorectomy of the intrahepatic metastasis was planned. For direct application of the 3D

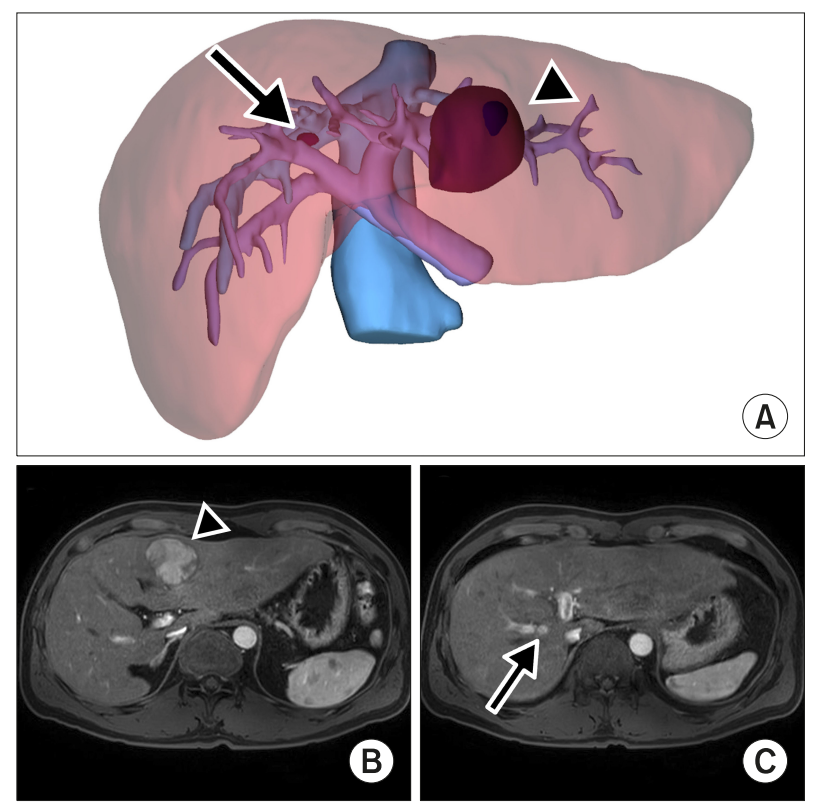

Fig. 1. Preoperative 3D modeling of the liver. (A) A 4.7 cm-sized hepatocellular carcinoma (arrowhead) was located on segment 3 and intrahepatic metastasis (arrow) located between the right anterior portal vein, middle hepatic vein and right hepatic vein. Preoperative magnetic resonance imaging shows (B) the main mass on segment 3 and (C) segment 8 right above right anterior portal vein. model during the surgery, 3D printing of the patient's anatomy was performed.

\section{Three-dimensional printed model for intraoperative guidance}

Based on the 3D modeled liver, additional editing was performed for practical application during the operation. Since we planned to perform a two-step procedure, we focused on the expected cut surface plane after left hemihepatectomy. The modeling procedure was performed by removing the structures that were unnecessary during the operation. The portal vein branches and middle and right hepatic veins that crossed through the region of interest were remained. Left liver and right liver beyond the region of interest was removed during modeling. For visualizing the inner structures within the liver, the hepatic parenchyma was removed while the boundaries were remained with a width of $1 \mathrm{~cm}$. Three-dimensional printing was done using Cubicon Single Plus (Cubicon, Seongnamsi, Korea). Fig. 2 shows modeling of the software and the actual 3D printed model.

\section{Intraoperative findings}

Under general anesthesia, right subcostal incision was made for open hepatectomy. Left hemihepatectomy was performed and the middle hepatic vein was preserved. Fig. 3A shows the comparison of the operative field after left hemihepatectomy and the 3D printed model. After comparing the printed model to the surgical field, dissection using cavitron ultrasonic surgical aspirator was done tracing above the right anterior glissonean pedicle. After tracing into the liver parenchyma, intrahepatic metastasis was identified and tumorectomy was done. (Fig. 3B) Fig. 3C shows the direct comparison between the printed model and the actual location of the tumor. Fig. 3D and $3 \mathrm{E}$ shows the surgical specimen of the patient. Three centimeter margin was obtained for the $4.7 \mathrm{~cm}$-sized main mass located on segment 3. The tumor grade was Edmondson grade II with a peritumoral microvascular invasion without portal vein invasion. Patient was discharged 14 days after the operation. Diuretics were prescribed due to post-hepatectomy ascites which resolved with medication. Although clean margin was obtained with the capsule preserved for the intrahepatic metastasis, adjuvant proton beam therapy was performed on the tumor bed of intra- 

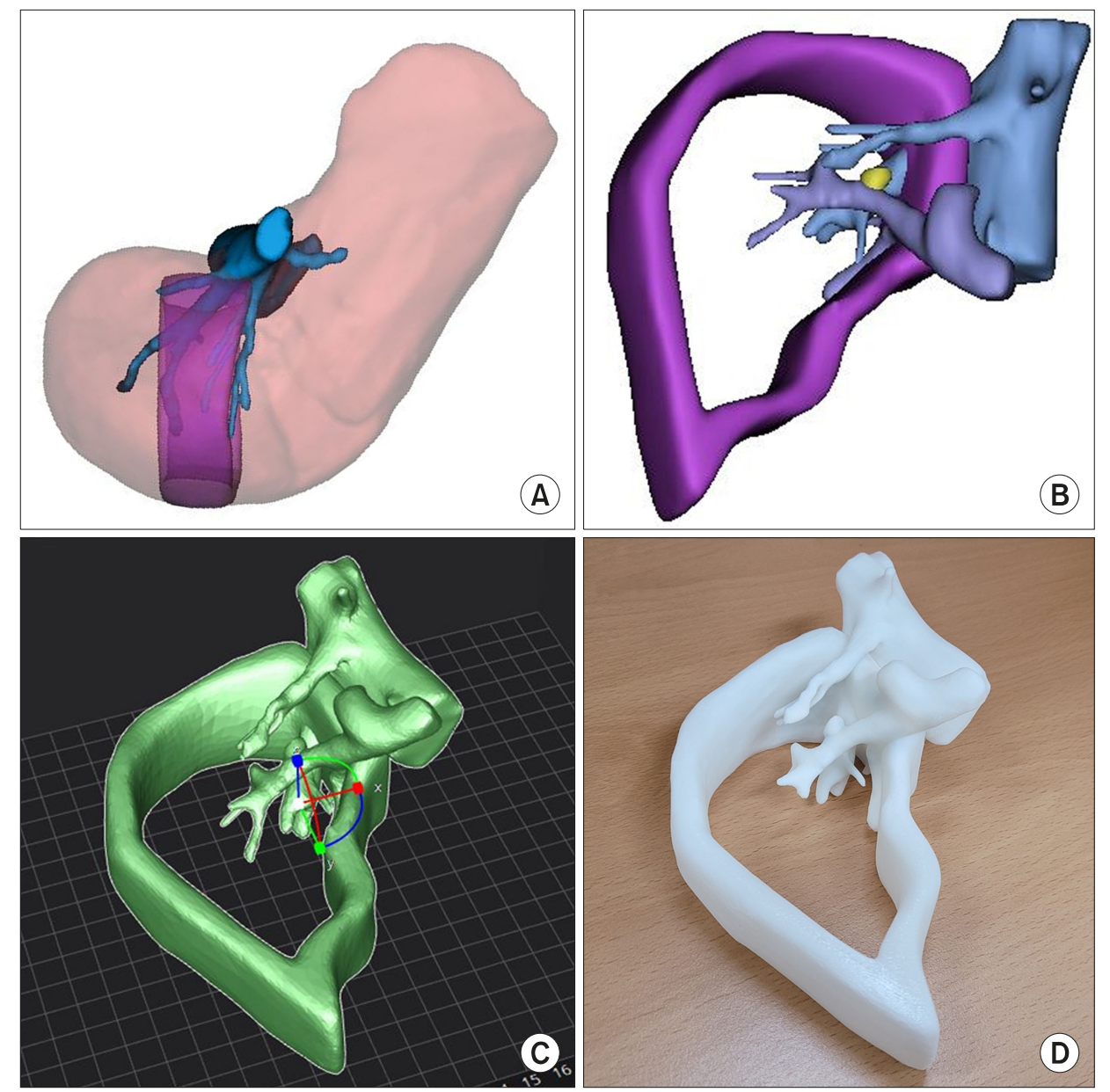

Fig. 2. Three-dimensional printing of the model. (A) The region of interest was set between the middle hepatic vein and 3 $\mathrm{cm}$ right side to the midline. (B) The liver parenchyma was removed to allow visualization of the inner vascular structure and intrahepatic metastasis. (C) The 3D model was edited (D) and printed using a $3 \mathrm{D}$ printer.

hepatic metastasis. The patient is under regular outpatient clinic follow up without recurrence.

\section{DISCUSSION}

Although 3D printing gained attention of medical professionals, the application in the field of surgery was limited. Bio printing using material such as polycarpolactone is used in reconstructive surgery involving bony structures. ${ }^{7}$ However, the application of $3 \mathrm{D}$ printing technology in abdominal surgery has been challenged since there is no bony structure that require reconstruction in the abdominal cavity. Most of the $3 \mathrm{D}$ printing cases that were used in liver surgery was individualized $3 \mathrm{D}$ models for preoperative planning. ${ }^{8}$ However, most liver surgeons are aware of the anatomy of liver based on conventional imaging modalities and therefore the practicality of 3D printing was limited only for education of their trainee and patient. The expensive cost for $3 \mathrm{D}$ printed liver was another barrier preventing its spread to the general field. Effort to reduce the cost and time for modeling and printing was also investigated by Japan investigators. ${ }^{9}$ Nevertheless, 3D printing technology is still not used sufficiently.

As a major center for liver resection and liver transplantation, surgeons of our team are trained in understanding the anatomy of the liver based on conventional imaging modalities. Therefore, 3D printing is not used routinely, and only applied on sophisticated cases. The case presented in this article raised some issues for treatment planning. It would have been best if there was a living donor for liver transplantation. Although the patient had two HCCs with one sizing up to $4.7 \mathrm{~cm}$, alpha fetoprotein level was relatively low. Since the patient had a cirrhotic liver, liver transplantation could offer definite treatment for both $\mathrm{HCC}$ and liver cirrhosis. The main mass was located in segment 3 and could safely be removed with left lateral sectionectomy. However, the intrahepatic metastasis was located on the most center of the 

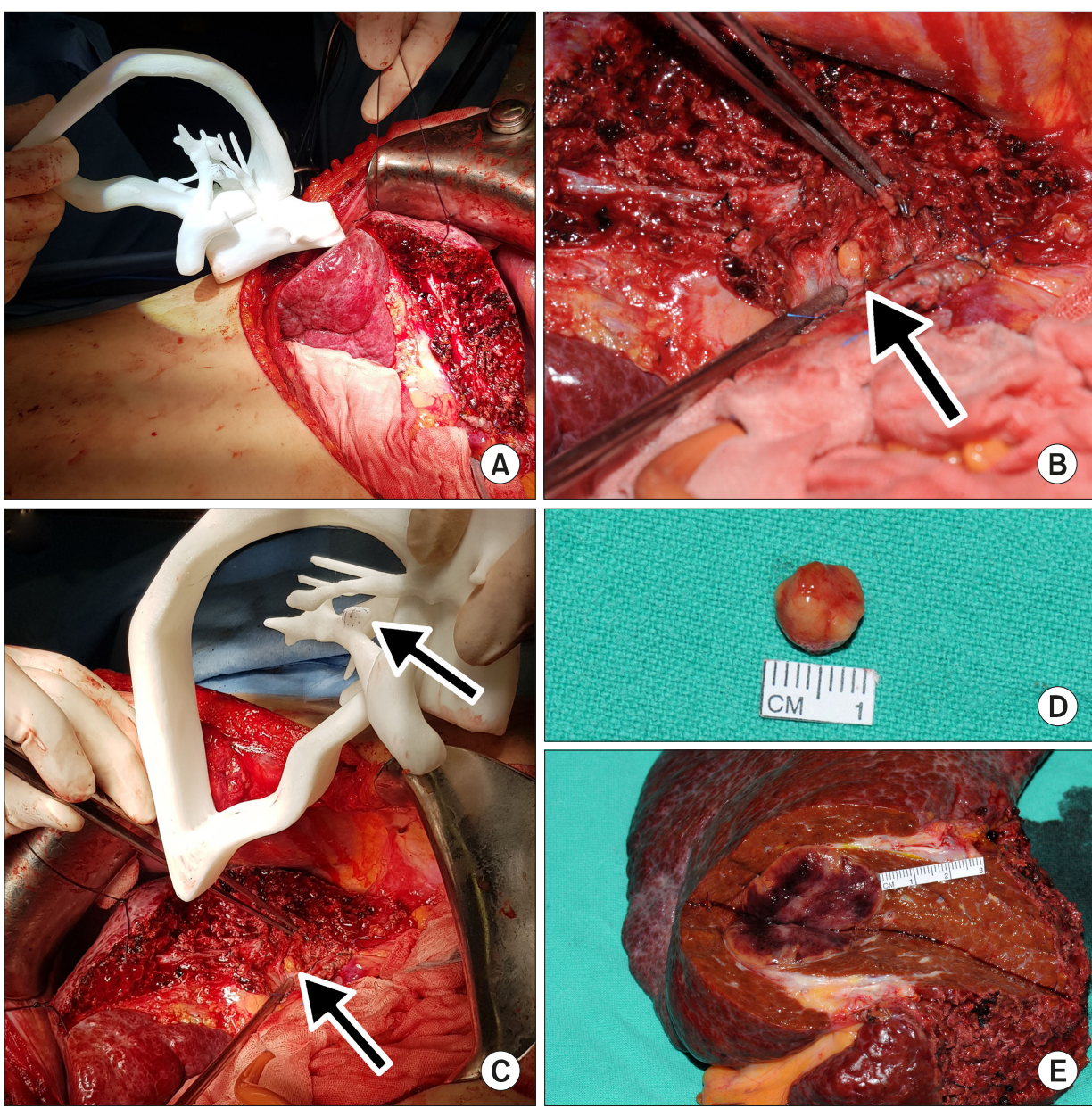

Fig. 3. Intraoperative finding during surgery. After left hemihepatectomy, (A) the 3D printed model was compared directly side by side as a guide. (B) After dissecting the liver parenchyma between the right anterior glissonean pedicle and middle hepatic vein, intrahepatic metastasis (arrow) was identified. (C) The 3D model was compared to the actual field with the intrahepatic metastasis exposed. (D) The intrahepatic metastasis was removed and (E) the main mass was removed with the left hemiliver with a $3 \mathrm{~cm}$ margin.

liver making it impossible for radiofrequency ablation. The tumor was located between the right anterior glissonean pedicle, middle hepatic vein and right hepatic vein. Performing extended left hemihepatectomy or left trisectionectomy could offer complete removal of the tumors. However, the left liver volume was enormously large and performing extended left hemihepatectomy would leave the patient with about 30 to $40 \%$ of functional future liver remnant.

Three-dimensional modeling allowed the precise understanding of the tumor location which was difficult to understand based on conventional imaging. Three-dimensional printing of the model offered direct visualization during operation. Our 3D-printed model only required 6 hours for printing and the material used for printing costed about 15 dollars. The size of the model was identical to the actual liver for directly comparing in the field. The printed and unprinted parts were tailored for efficient printing and visualization. The limitation of this case re- port is that the technique we used cannot be generalized to other liver surgery cases. The printed model was efficiently modeled by professional biomedical artist who was aware of the surgical field and liver anatomy. Nevertheless, we believe that our case can offer opportunities for certain surgeons with a need for better understanding of difficult cases.

\section{ACKNOWLEDGEMENTS}

This research was supported by the Basic Research Program through the National Research Foundation of Korea (NRF) funded by the Ministry of Science and ICT (2020R1C1C1010525).

\section{CONFLICT OF INTEREST}

There are no conflicts of interest related to the article. 


\section{ORCID}

Jinsoo Rhu: https://orcid.org/0000-0001-9809-8525

Mi Seung Kim: https://orcid.org/0000-0003-0621-2007

Sangjin Kim: https://orcid.org/0000-0002-0080-176X

Gyu-Seong Choi: https://orcid.org/0000-0003-2545-3105

Jong Man Kim: https://orcid.org/0000-0002-1903-8354

Jae-Won Joh: https://orcid.org/0000-0003-1732-6210

\section{AUTHOR CONTRIBUTIONS}

Conceptualization: JR, MSK. Methodology: JR, SK, GSC, JMK, JWJ. Project administration: JR, GSC, JMK, JWJ. Visualization: JR, MSK, SK, GSC, JMK. Writing original draft: JR. Writing - review \& editing: JR, GSC, JMK, JWJ.

\section{REFERENCES}

1. Ercolani G, Grazi GL, Ravaioli M, Del Gaudio M, Gardini A, Cescon $\mathrm{M}$, et al. Liver resection for hepatocellular carcinoma on cirrhosis: univariate and multivariate analysis of risk factors for intrahepatic recurrence. Ann Surg 2003;237:536-543.

2. Fong Y, Sun RL, Jarnagin W, Blumgart LH. An analysis of 412 cases of hepatocellular carcinoma at a Western center. Ann Surg 1999;229:790-799; discussion 799-800.

3. Rustgi VK. Epidemiology of hepatocellular carcinoma. Gastroenterol Clin North Am 1987; 16:545-551.

4. Grazi GL, Ercolani G, Pierangeli F, Del Gaudio M, Cescon M, Cavallari A, et al. Improved results of liver resection for hepatocellular carcinoma on cirrhosis give the procedure added value. Ann Surg 2001;234:71-78.

5. Regimbeau JM, Kianmanesh R, Farges O, Dondero F, Sauvanet A, Belghiti J. Extent of liver resection influences the outcome in patients with cirrhosis and small hepatocellular carcinoma. Surgery 2002;131:311-317.

6. Rhu J, Kim SJ, Choi GS, Kim JM, Joh JW, Kwon CHD. Laparoscopic versus open right posterior sectionectomy for hepatocellular carcinoma in a high-volume center: a propensity score matched analysis. World J Surg 2018;42:2930-2937.

7. Louvrier A, Marty P, Barrabé A, Euvrard E, Chatelain B, Weber E, et al. How useful is $3 \mathrm{D}$ printing in maxillofacial surgery? $\mathrm{J}$ Stomatol Oral Maxillofac Surg 2017;118:206-212.

8. Witowski JS, Coles-Black J, Zuzak TZ, Pędziwiatr M, Chuen J, Major $\mathrm{P}$, et al. 3D printing in liver surgery: a systematic review. Telemed J E Health 2017;23:943-947.

9. Kuroda S, Kobayashi T, Ohdan H. 3D printing model of the intrahepatic vessels for navigation during anatomical resection of hepatocellular carcinoma. Int J Surg Case Rep 2017;41:219-222. 